\title{
Corneal Limbal Stem Cell Cryopreservation
}

\begin{abstract}
Keywords: Cryopreservation; Limabl stem cell; Comea
Abstract

Background: Limbal stem cell therapy has been used successfully to treat various comeal disorders. Cryopreservation of limbal stem cells for the purpose of tissue banking can provide great benefits for eye patients. Although the cryopreservants for storing limbal tissues have been developed previously, the characteristics of the limba stem cells derived from the cryopresenved tissues were unclear, and the cell viability of limbal expansion on amniotic membranes (AMN) after cryopreservation was $\sim 50 \%$. In this study, we have applied a different culture procedure for the limbal stem cell expansion and cryopreservation to explore the feasibility of using cryopreserved limbal tissues for stem cell therapy.
\end{abstract}

Methods: Comeolimbal tissue discs were dissected from human donor comeas, and explant cultures were performed on plastic or AMN. The limbal expansions were maintained in monolayer cultures (without a ir-lifting) for two weeks before harvest or cryopreservation, which is similar to the procedure used for the autologous transplants in humans. To investigate the effects of cryopreservation on limbal stem cell outgrowth, (1) dissected limbal tissue from each donor was also stored in liquid nitrogen with cryoprotectants prior to explant culture; (2) Limbal stem cells expanded on AMN were cryopreserved and subsequently revived to evaluate their viability after freezing and thawing by propidium iodide staining.

Results: Both cryopreserved limbal tissues and limbal stem cells cultured on AMN displayed robust outgrowth after tha wing. The limba expansion on AMN remained viable after cryopreservation (87.5\% cell survival). The limbal explant cultures exhibited strong positive staining for $A B C G 2, \triangle N p 63$ and vimentin (stem cell markers), but weak and sporadic staining for $\mathrm{K} 3$ (a differentiation marker). Western blots and RT-PCR experiments confirmed the expression of these cell markers.

Conclusion: Proper cryopreservation of comeal limbal tissues can be an effective method for preserving limbal stem cells and for expansion on amniotic membranesfortransplantation at a later date.

\section{Abbreviations}

DMSO: dimethyl sulfoxide; AMN, amniotic membrane; PI: propidium iodide

\section{Introduction}

The corneal limbus has been shown to be rich in putative corneal stem cells [1]. Being essential for corneal tissue integrity and function, these limbal stem cells have great potential for therapeutic applications in individuals with certain corneal pathologies. Transplantion of limbal stem cells and amniotic membranes (AMN) have greatly facilitated ocular surface reconstructions, which are widely used to treat thermal and chemical burns, injuries from radiation, severe infection, ocular pemphigoid, Stevens-Johnson syndrome and other corneal disorders. The autologous transplantation of human limbal stem cells expanded on AMN in vitro has become one of the most effective methods to restore useful vision in patients suffering from various corneal injuries and diseases [2].

While either a patient's biopsy tissue or donor corneal tissue preserved in Optisol GS provides reliable sources of limbal tissue for the in vitro expansion of limbal stem cells, recent studies have

\section{Journal of}

\section{Ocular Biology}

\author{
Jillian Ewel, Ching Yuan, Elizabeth F. Nelson, and \\ Stephen C. Kaufman* \\ Department of Ophthalmology, University of Minnesota, \\ Minneapolis, Minnesota, USA \\ *Address for Correspondence \\ Stephen C. Kaufman, M.D., Ph.D., Department of Ophthalmology, \\ University of Minnesota, Phillips Wangensteen Building Room 9-241, \\ 516 Delaware Street S.E., Minneapolis, MN, USA, Tel: +1(612)625-5413; \\ Fax: +1(612)626-4455; E-mail: sck@umn.edu \\ Copyright: () 2013 Ewel J, et al. This is an open access article \\ distributed under the Creative Commons Attribution License, which \\ permits unrestricted use, distribution, and reproduction in any medium, \\ provided the original work is properly cited. \\ Submission: 30 July 2013 \\ Accepted: 29 August 2013 \\ Published: 02 September 2013 \\ Reviewed \& Approved by: Dr. Qizhou Lian \\ Department of Medicine and Ophthalmology \\ The University of Hong Kong, China
}

explored the feasibility of using cryopreservation to extend the shelf life of these precious tissues. This would allow for multiple transplants, as in cases of failed surgery or surgical complications, without requiring multiple biopsies or donor tissues. Cryopreserved limbal tissues from donor corneas have been shown to resume robust outgrowth after storage in liquid nitrogen for 2 to 12 months [3]. Limbal stem cells harvested from explant cultures then cryopreserved were successfully revived and used for transplantation in rabbit and goat models [4-6]. Furthermore, when compared to cryopreservation of limbal stem cells after one week, 8 weeks of cryopreservation in liquid nitrogen did not significantly decrease the viability of limbal stem cells grown on AMN, despite only 54\% cell survival [7]; in the same report, limbal stem cells subjected to cryopreservation retained expression of ABCG2 and vimentin, two markers used to charactize limbal stem cells. Future endeavors to optimize cryopreservation conditions and better preserve the proliferation potential of limbal stem cells and their "stemness" will provide great clincal benefits to ophthalmic patients

In the current study, we have further characterized the effects of cryopreservation on limbal tissues and limbal stem cells expanded on AMN, using the previously reported cryopreservants $[3,7]$ but with a different culture procedure. Using immunostainings, western blots, and reverse transcription PCR (RT-PCR) techniques, we confirmed that cells growing out of cryopreserved limbal tissues express limbal stem cell markers under our culture conditions. Propidium iodide (PI) staining was used to evaluate the cell death of cryopreserved limbal stem cells grown on AMN after revival. Our results contribute to the growing body of research that supports the potential role of cryopreservation in advancing limbal stem cell therapy.

\section{Materials and Methods}

\section{Preparation of denuded amniotic membrane}

AMN (Bio-Tissue, Inc., Miami, FL) were treated with $4 \mathrm{mg} / \mathrm{mL}$ dispase II (Roche Applied Science, Indianapolis, IN) for one hour at $37^{\circ} \mathrm{C}$. The epithelia were then manually removed using a rubber policeman and rinsed in 1x Dulbecco's PBS (Gibco, Invitrogen Corp., Carlsbad, CA) before use. 


\section{Preparation and culture of limbal stem cells}

Donor cornea buttons were obtained from Minnesota Lion's Eye Bank, Minneapolis, MN, with Institutional Review Board exemption. Corneas from 12 donors (a total of 24 corneas) were used in this study, with ages ranging from 52 to 75 , except one 18 years old. The 18 year old donor cornea was only used and included in the PI staining of limbal stem cells expanded on AMN. Two millimeter corneolimbal discs were dissected from donor corneal tissue. The corneolimbal discs were bisected; half of the tissue blocks from each donor were cultured immediately for controls, and the other half were cryopreserved before being cultured. The limbal tissue blocks were handled with great care and the explant cultures of limbal stem cells from each donor were performed on either glass chamber slides for immunostaining analysis, or on $1 \mathrm{x} 3 \mathrm{~cm}$ sections of denuded AMN for immunostaining and PI analysis; tissue from each donor was also cultured on $100 \mathrm{~mm}$ plastic culture dishes for either RNA extraction or harvesting cell lysates. Cultures were maintained at $37^{\circ} \mathrm{C}$ in a humidified atmosphere containing $5 \% \mathrm{CO}_{2}$, and the medium was changed every 2 days. The limbal expansions were maintained in monolayer cultures (without air-lifting) for two weeks before harvest or cryopreservation, which is similar to the procedure used for the autologous transplants in humans [2]. The culture medium consisted of Dulbecco's modified Eagle's medium (DMEM)/Ham's F12 (Cellgro, Mediatech, Inc., Manassas, VA) supplemented with 10\% fetal bovine serum (FBS; Atlanta Biologicals, Lawrenceville, GA); $1 \mathrm{x}$ insulin-transferrin-sodium selenite supplement (Sigma-Aldrich, Saint Louis, MO); $0.5 \mu \mathrm{g} / \mathrm{mL}$ hydrocortisone (Sigma-Aldrich, Saint Louis, MO), $2 \mathrm{ng} / \mathrm{mL}$ recombinant human epidermal growth factor (Gibco, Invitrogen Corp., Carlsbad, CA), 0.5\% dimethyl sulfoxide (DMSO; Sigma-Aldrich, Saint Louis, MO), $50 \mu \mathrm{g} / \mathrm{mL}$ penicillin/ streptomycin (Gibco, Invitrogen Corp., Carlsbad, CA), $1.25 \mu \mathrm{g} / \mathrm{mL}$ amphotericin B (Sigma-Aldrich, Saint Louis, MO), and $0.1 \mu \mathrm{g} / \mathrm{mL}$ epinephrine (Sigma-Aldrich, Saint Louis, MO).

\section{Cryopreservation of limbal tissues and limbal stem cell cultures}

Limbal tissue: Tissue blocks were placed in $1.5 \mathrm{~mL}$ cryotubes containing $1 \mathrm{~mL}$ cryopreservation medium composed of 70\% Optisol GS (Chiron Ophthalmics, Inc., Irvine, CA), 20\% FBS, and 10\% DMSO [3]. The cryotubes were then placed in a CellFreezer (Research Products International, Corp., Mount Prospect, IL) containing a isopropanol bath for controlled-rate freezing, and stored at $-80^{\circ} \mathrm{C}$ overnight before being moved to liquid nitrogen storage. After four weeks in storage, sample tubes were removed from liquid nitrogen and thawed rapidly in a $37^{\circ} \mathrm{C}$ water bath. Tissue blocks were then removed from the cryopreservation medium and used for explant culture per the protocol above.

Limbal stem cell cultures on AMN: Limbal explant cultures were obtained by culturing limbal tissue blocks on AMN for two weeks. At this point, samples were divided in half. While one half was used immediately for immunostaining, the other was cryopreserved prior to analysis: limbal stem cells and the AMN on which they were growing were preserved in cryopreservation medium composed of $60 \%$ DMEM/Ham's F12, 30\% FBS, and 10\% DMSO [7], frozen in a CellFreezer as mentioned above, then stored in liquid nitrogen for four weeks. Cell cultures on AMN were removed from liquid nitrogen and rapidly thawed in a $37^{\circ} \mathrm{C}$ water bath as for tissue samples above, then incubated in growth medium for one hour at $37^{\circ} \mathrm{C}$ prior to PI staining.

\section{Immunofluorescent staining of explant cultures for limbal stem cell markers}

Explant cultures grown on glass chamber slides were analyzed for limbal stem cell markers by immunohistochemistry. After fixation and blocking, limbal stem cell cultures were incubated in either mouse antibody against ABCG2, clone BXP-21 (Sigma-Aldrich, Saint Louis, MO); mouse antibody against cytokeratin K3/K76, clone AE5 (Progen Biotechnik, Heidelberg, Germany); mouse antibody against p63, isoform $\Delta \mathrm{Np63}$, clone 4A4 (Sigma-Aldrich, Saint Louis, MO); or mouse antibody against vimentin, clone V9 (Sigma-Aldrich, Saint Louis, MO); followed by secondary antibody Alexa Flour ${ }^{\circledR} 488$ (Molecular Probes, Invitrogen Corp., Carlsbad, CA). Cell nuclei were counter-stained with Hoechst 33342 dye. Slides were mounted with cover slips and analyzed for signal detection by a Zeiss Axiovert 200M epi-fluorescence microscope.

Western blot analysis of expression of limbal stem cell markers

Cell lysates from limbal explant cultures were harvested and then separated by SDS-PAGE gel electrophoresis, followed by Western transfer. Blots were probed for $\mathrm{p} 63$ and vimentin with the same antibodies as were used for immunohistochemistry analyses; IRDye ${ }^{\mathrm{Tu}}$ 700 anti-mouse IgG (LI-COR Biosciences, Lincoln, NE) was used for secondary antibody. An Odyssey ${ }^{\circ}$ Infrared Imaging System (LI-COR Biosciences, Lincoln, NE) was used to image blots, which were then stripped with $25 \mathrm{mM}$ glycine, $2 \% \mathrm{SDS}(\mathrm{pH} \mathrm{2.0)}$ ) and reprobed with mouse antibody against $\beta$-actin, clone AC-15 (Sigma-Aldrich, Saint Louis, MO).

RT-PCR and real-time RT-PCR (qRT-PCR) analysis of mRNA expression of limbal stem cell markers

Total RNA was extracted from limbal explant cultures grown on plastic culture dishes using an RNeasy ${ }^{\circ}$ Mini Kit (QIAGEN, Inc., Valencia, CA) per the manufacturer's instructions; an RNasefree DNase I Set (QIAGEN, Inc., Valencia, CA) was used to digest genomic DNA. Total RNA (5 $\mu \mathrm{g}$ ) was reverse-transcribed using a Superscript III First-Strand Synthesis System (Invitrogen Corp., Carlsbad, CA) according to the manufacturer's instructions. PCR was performed on a RoboCycler ${ }^{\circledR}$ Gradient 96 thermal cycler (Stratagene, Corp., Agilent Technologies, Inc., Santa Clara, CA) with Choice ${ }^{\text {Tax }}$ Taq DNA Polymerase in the supplied reaction buffer (Denville Scientific, Inc., Metuchen, NJ) and primers for ABCG2, vimentin, p63, cytokeratin K3, and $\beta$-actin (Table 1). Thirty-three cycles were used to amplify target cDNA in $50 \mu \mathrm{L}$ reactions, with each cycle consisting of denaturation for $45 \mathrm{~s}$ at $95^{\circ} \mathrm{C}$, annealing for $30 \mathrm{~s}$ at annealing temperature (Table 1), and elongation for $30 \mathrm{~s}$ at $72^{\circ} \mathrm{C}$. Products were separated by electrophoresis on $1.2 \%$ agarose gels containing ethidium bromide and visualized under UV light. Real-time PCR was performed on an iCycler thermal cycler (Bio-Rad Laboratories, Inc., Hercules, CA) with iQ SYBR Green Supermix (Bio-Rad Laboratories, Inc., Hercules, CA) and primers for ABCG 2 and $\beta$-actin (Table 1). An $\mathrm{iQ}^{\mathrm{s}} 5$ real-time PCR detection system and accompanying $\mathrm{iQ}^{\mathrm{m}} 5$ optical system software (Bio-Rad Laboratories, Inc., Hercules, CA) were used to capture and analyze qRT-PCR data from $25 \mu \mathrm{L}$ reactions run for 35 amplification cycles; each amplification cycle consisted of $15 \mathrm{~s}$ at $95^{\circ} \mathrm{C}$ 
Table 1: The sequences of oligonucleotide primers used in the RT-PCR experiments.

\begin{tabular}{|c|c|c|c|c|}
\hline Gene Accession Number & Primer & Sequence (5'-3') & $\begin{array}{l}\text { Size of PCR product } \\
\text { (base pairs) }\end{array}$ & $\begin{array}{c}\text { Annealing } \\
\text { temperature }\left({ }^{\circ} \mathrm{C}\right)\end{array}$ \\
\hline \multirow[t]{2}{*}{ NM_004827.2 } & ABCG2_F & GAGCCTACAACTGGCTTAGACTCAA & 85 & 60 \\
\hline & ABCG2_R & TGATTGTTCGTCCCTGCTTAGAC & & \\
\hline \multirow[t]{2}{*}{ NM_057088.2 } & K3_F & GGCAGAGATCGAGGGTGTC & 145 & 57 \\
\hline & K3_R & GTCATCCTTCGCCTGCTGTAG & & \\
\hline \multirow[t]{2}{*}{ NM_001114982.1 } & p63_F & GGAGCCAGAAGAAAGGACAGCAGC & 128 & 67 \\
\hline & p63_R & GGTCCATGCTGTTCAGGAGCCC & & \\
\hline \multirow[t]{2}{*}{ NM_003380.3 } & Vimentin_F & CCTTGAACGCAAAGTGGAATC & 106 & 57 \\
\hline & Vimentin_R & GACATGCTGTTCCTGAATCTGAG & & \\
\hline \multirow[t]{2}{*}{ NM_001101.3 } & $\beta$-actin_F & TGTCCACCTTCCAGCAGATGT & 90 & 60 \\
\hline & $\beta$-actin_R & AGTCCGCCTAGAAGCATTTGC & & \\
\hline
\end{tabular}

for denaturation and $1 \mathrm{~min}$ at $60^{\circ} \mathrm{C}$ for annealing and elongation [8]. The $\Delta \mathrm{C}_{\mathrm{T}}$ method was used to calculate the fold change expression of ABCG2 normalized to $\beta$-actin.

\section{Propidium iodide staining of cyropreserved limbal stem cell cultures}

Propidium iodide (PI) staining was performed on limbal stem cell cultures expanded on denuded AMN, both before and after cryopreservation, by incubating cultures for 5 minutes in serumfree medium (SFM) containing PI. After incubation in the stain, cultures were washed extensively with SFM, and then AMN were carefully removed from their nitrocellulose backing to be laid flat on microscope slides. A Zeiss Axiovert $200 \mathrm{M}$ epi-fluorescence microscope was used to detect PI signal, and the number of dead cells were determined by counting PI-positive cells. Cells within the same viewing area were then lysed with $1 \%$ Triton X-100, washed with SFM, and stained again with PI to determine total cell numbers and thus estimate the percentage of cell death.

\section{Results}

Characterization of limbal explant cultures from cryopreserved limbal tissue blocks

After storage for four weeks in liquid nitrogen, limbal tissue discs were revived and cultivated for limbal explant culture. Robust outgrowth from these cryopreserved tissues was observed within 48 hours, which was similar to the period in which outgrowth appeared from control limbal tissues that were freshly cultivated without cryopreservation. Immunofluorescence staining of control limbal explant cultures showed strong signals for the limbal stem cell markers ABCG2, p63 and vimentin; and either no signal or very weak signal for differentiation markers of epithelial cells (cytokeratin K3, Figure 1, and cytokeratin K12, data not shown). Immunostaining results of explant cultures from cryopreserved limbal tissue exhibited similar signals to controls (Figure 1, "After Cryopreservation").

RT-PCR was used to amplify mRNA transcripts of the four aforementioned markers (Figure 2A). Comparable results for $\mathrm{p} 63$ and vimentin were also obtained by Western blots (Figure 2B), whereas K3 was not detected from the cell lysate likely due to its low expression level (Figure 2C). Along with RT-PCR, quantitative PCR was used to compare expression levels of ABCG2 mRNA before and after cryopreservation (Figure 3). Although not being statically significant,
ABCG2 expression in limbal stem cell expansion cryopreserved before culture was higher, when compared to control samples from stem cells expanded from freshly cultured limbal tissues.

\section{Viability of cryopreserved limbal stem cells expanded on} amniotic membrane

In addition to cryopreserving limbal tissue discs for later stem cell expansion, we also investigated cryopreserving limbal stem cells on AMN. Limbal stem cell cultures established as monolayers on AMN were cryopreserved for four weeks in liquid nitrogen then revived; within one day of being revived, limbal stem cells at the edges of AMN were observed by light microscopy to have proliferated beyond the membrane (data not shown). The viability of these cryopreserved limbal stem cells was examined by PI staining. Very few limbal stem cells expanded on AMN stained positive with PI before cryopreservation (2.4\% on average), indicating high cell viability and minimal cell death (Figure 4A and 4C); PI stainings of limbal stem cells on AMN after cryopreservation showed more positive

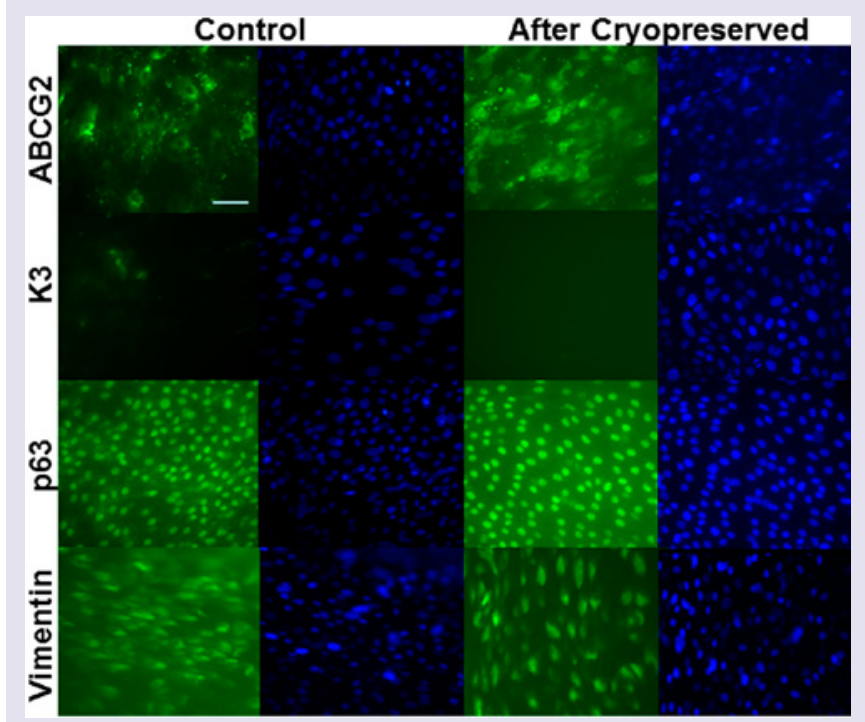

Figure 1: Characterization of limbal explant cultures by immunofluorescent staining. Cells were stained with antibodies specific for ABCG2, K3, p63 and vimentin (FITC). Nuclei counterstained with Hoechst 33342 (DAPI). Scale bar $=100 \mu$. 
staining $(12.5 \%$ on average, Figure $4 \mathrm{~B}$ and $4 \mathrm{D})$, and quantification of these results verified that this increase in cell death was significant (Figure 4E). However, while the difference in cell death was significant between control and cryopreserved cells, the PI staining results demonstrated that the majority of limbal stem cells $(87.5 \%)$ remained viable following cryopreservation. RT-PCR also confirmed the expression of ABCG2 in the cryopreserved limbal stem cells expanded on the denuded AMN (data not shown).

\section{Discussion}

Previous studies have supported the feasibility of cryopreserving limbal tissues for explant culture [3,5-7,9]. Cryopreservation of
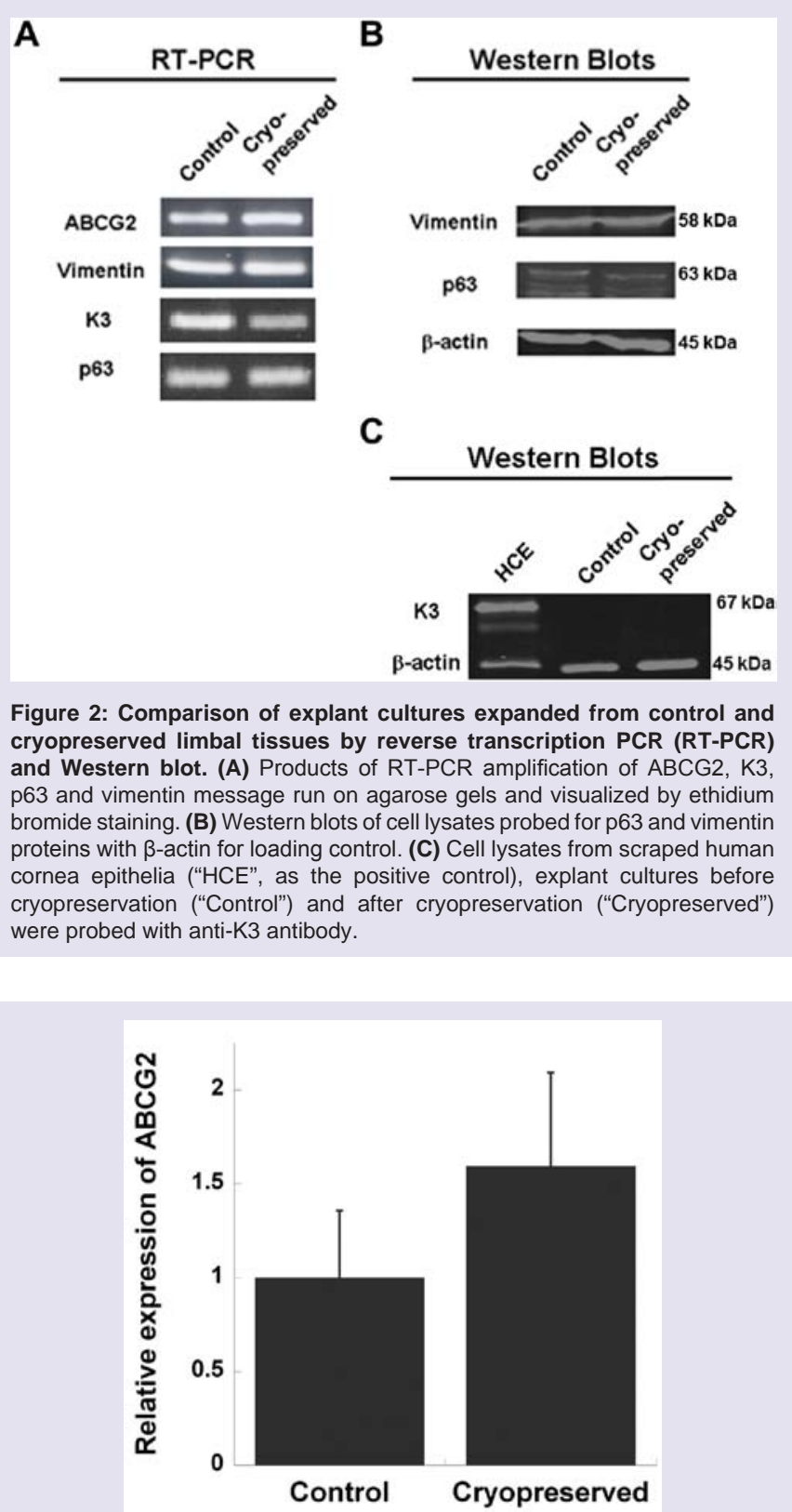

Figure 3: qRT-PCR analysis of the relative expression of ABCG2. Human limbal samples were compared for the expression of ABCG2 gene before and after cryopreservation $(n=3$, bar=SEM, $p=0.22)$.
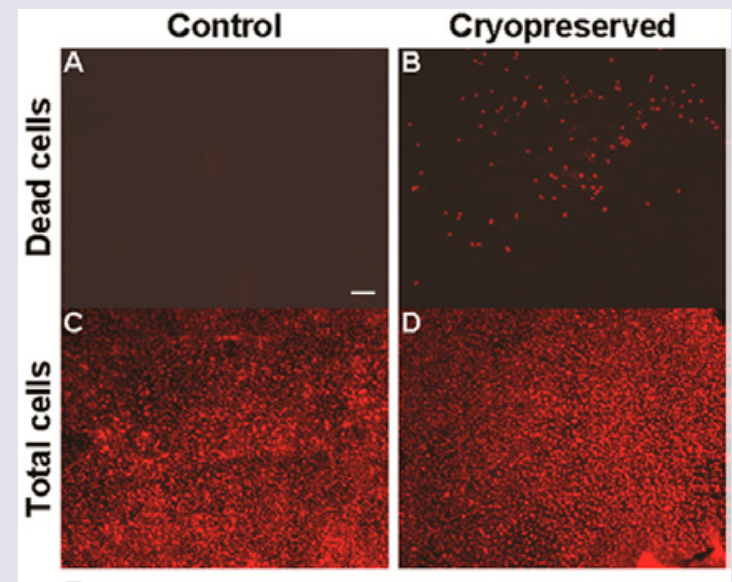

$E_{20}$

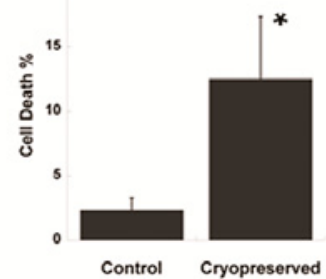

Figure 4: Cell death of the cryopreserved limbal tissues after thawing Propidium iodide $(\mathrm{PI})$ stainings of limbal stem cells expanded on amniotic membranes before (A) and after (B) cryopreservation. (C) and (D) Total cells stained with PI following permeation with detergent. 100x magnification. (E) Quantification of cell death expressed as percentage of total cells. ( $n=6$, bar=SEM, $p=0.007)$ Scale bar $=150 \mu$

donor tissues would allow tissue banks to keep transplantable donor tissue for longer periods, increasing tissue placement and decreasing waste of quality tissues. Furthermore, by preserving limbal stem cells cultivated on AMN from a patient's biopsy tissue, a clinician would be able to perform multiple autologous transplants, if necessary, without putting the patient through multiple biopsies. Thus, cryopreserved limbal stem cells have the potential to provide new and better treatment options to patients with corneal surface pathology.

Our results demonstrate that ex vivo expansion of cryopreserved limbal stem cells retain their proliferation potential, confirming previous results [3]. While Bratanov and colleagues reported the presence of "lymphoblast-like" cells with morphological features similar to limbal stem cells or to transient amplifying cells described by others [4], the immunostainings of expanded cell cultures were largely negative for limbal stem cell markers p63 and vimentin [3]. This negative result may be due to the prolonged culture time (28 days) used in their study, which can lead to corneal stem cell differentiation. In contrast, our current study clearly demonstrated that the two-week explant cultures expanded from the cryopreserved limbal tissues retained their stem cell characteristics, as demonstrated by strong immunofluorescent staining of not only p 63 and vimentin, but also ABCG2. This expression profile has been previously shown to successfully identify and characterize limbal stem cells [1]. ABCG2, a member of the ATP binding cassette transporters, was identified originally for its ability to produce drug resistance in cancer cells and later as a primitive stem cell marker; later studies found it is expressed in the basal layer of the limbal epithelium but not in the cornea [10]. Similarly, p63 is localized to the limbus region and is a putative marker 
for limbal stem cells. Specifically, the isoform $\Delta \mathrm{Np} 63 \alpha$ is localized to the limbal basal layer and thought to be the identifier of limbal stem cells [11]. Vimentin has been shown to be localized to the limbuscornea border, with particularly high expression in the basal limbal cells [3]; while this marker alone does not definitively identify limbal stem cells, co-expression of vimentin with ABCG2 and p63 increases the likelihood that those cells still retain their stem cell properties.

Immunohistochemical analyses of our limbal explant cultures also revealed minimal expression of cytokeratin K3, a differentiation marker expressed by differentiated corneal epithelial cells. Lack of K3 expression in the corneal epithelial cultures indicates cells that are undifferentiated, a characteristic of limbal stem cells [12]. Although the absence of a single differentiation marker cannot solely identify stem cells, when included with positive ABCG2, p63 and vimentin results, a negative $\mathrm{K} 3$ result supports evidence for the presence of limbal stem cells.

RT-PCR and Western blots results corroborated immunostaining results. Since ABCG2 is likely the best marker of limbal stem cells, we also used qRT-PCR to compare expression of ABCG2 mRNA in stem cells expanded from freshly cultured limbal tissues and in stem cells expanded from limbal tissues cryopreserved before culture. Our results showed that there was an increase in the expression of ABCG2 even though this difference is not statistically significant. One explanation for the observed results is that the cryopreservation process causes the up-regulate of ABCG2 expression in limbal stem cells. ABCG2 has previously been shown to play an anti-oxidative role in corneal epithelial cells, suggesting it aids in protection against oxidative stress [13]. This gene may therefore play a role in protecting limbal stem cells from stress related to the freeze-thaw process. Another possibility is that differentiated epithelial cells may be more vulnerable to the cryopreservation process than the undifferentiated limbal stem cells, and contribute to the observed cell death after cryopreservation.

Previously, it was found that limbal stem cells expanded on AMN can be successfully cryopreserved and revived for further outgrowth, but the viability of these revived cells (54\% cell survival) was considerably low [7]; however, low cell viability may not be an issue since the surviving cells seemed to retain their proliferation potential and quickly repopulated. We found that limbal cells grown on AMN as monolayers without air-lifting and cryopreserved have a high viability ( $87.5 \%$ cell survival), and their expression of ABCG2 suggests these cells also retain their stem cell characteristics. The significant discrepancy in cell viability between our results and previous findings may be attributable to a difference in culture methods: while Yeh et al. used an air-lifting procedure to culture their limbal cells, therefore creating stratified epithelia, we used a submersive technique similar to Tsai et al. to cultivate as monolayered explant cultures for autologous transplantation in humans. Another possible explanation may be the use of different assays to assess cell viability versus cell death $[2,7]$. Yeh et al. used an assay that measures cellular metabolism to derive the cell viability indirectly from an established standard curve [7]. Since the assay was performed right after the cells were thawed, cell viability may have been underestimated as cells may not have completely recovered from the freeze-thaw process and thus may have had compromised metabolisms. On the other hand, the PI staining method we used relies on the dye permeating the cell membrane of dying or dead cells so that these cells may be counted individually. Because DMSO (present in the cryopreservation medium) increases membrane permeability, we performed PI stainings after cells were revived and had incubated in growth medium for at least one hour. Regardless of the difference in cell viability, both studies demonstrate that limbal stem cells expanded on AMN will continue their robust outgrowth even after cryopreservation.

In summary, using modified procedures adapted from previous cryopreservation studies, we have confirmed the feasibility of cryopreserving two sources of limbal stem cells: donor limbal tissues and limbal explant cultures expanded on AMN. Although Bratanov et al. and Yeh et al. did not find significantly different results comparing 4 weeks to 8 weeks or longer cryopreservation times, our future research will examine the effect of longer cryopreservation times on stem cell viability $[3,7]$. Furthermore, to confirm the therapeutic potential of these stem cells, future studies with animal transplantation models need to assess the effectiveness of cryopreserved limbal stem cells in treating ocular surface disorders.

\section{References}

1. Schlötzer-Schrehardt U, Kruse FE (2005) Identification and characterization of limbal stem cells. Exp Eye Res 81: 247-264.

2. Tsai RJ, Li LM, Chen JK (2000) Reconstruction of damaged corneas by transplantation of autologous limbal epithelial cells. N Engl J Med 343: 86-93.

3. Bratanov M, Neronov A, Nikolova E (2009) Limbal explants from cryopreserved cadaver human corneas. Immunofluorescence and light microscopy of epithelial cells growing in culture. Cryo Letters 30: 183-189.

4. Kito K, Kagami H, Kobayashi C, Ueda M, Terasaki H (2005) Effects of cryopreservation on histology and viability of cultured corneal epithelial cell sheets in rabbit. Cornea 24: 735-741.

5. Mi S, Yang X, Zhao Q, Qu L, Chen S, et al. (2008) Reconstruction of corneal epithelium with cryopreserved corneal limbal stem cells in a goat model. Mol Reprod Dev 75: 1607-1616.

6. Oh JY, Kim MK, Shin KS, Shin MS, Wee WR, et al. (2007) Efficient cryopreservative conditions for cultivated limbal and conjunctival epithelial cells. Cornea 26: 840-846.

7. Yeh HJ, Yao CL, Chen HI, Cheng HC, Hwang SM (2008) Cryopreservation of human limbal stem cells ex vivo expanded on amniotic membrane. Cornea 27: 327-333.

8. Zhang W, Mojsilovic-Petrovic J, Andrade MF, Zhang H, Ball M, et al. (2003) The expression and functional characterization of ABCG2 in brain endothelial cells and vessels. FASEB J 17: 2085-2087.

9. Vasania VS, Prasad P, Gill RK, Mehta A, Viswanathan C, et al. (2011) Molecular and cellular characterization of expanded and cryopreserved human limbal epithelial stem cells reveal unique immunological properties. Exp Eye Res 92: 47-56

10. de Paiva CS, Chen Z, Corrales RM, Pflugfelder SC, Li DQ (2005) ABCG2 transporter identifies a population of clonogenic human limbal epithelial cells. Stem Cells 23: 63-73.

11. Di Iorio E, Barbaro V, Ruzza A, Ponzin D, Pellegrini G, et al. (2005) Isoforms of DeltaNp63 and the migration of ocular limbal cells in human corneal regeneration. Proc Natl Acad Sci USA 102: 9523-9528.

12. Proulx S, d'Arc Uwamaliya J, Carrier P, Deschambeault A, Audet C, et al (2010) Reconstruction of a human cornea by the self-assembly approach of tissue engineering using the three native cell types. Mol Vis 16: 2192-2201.

13. Kubota M, Shimmura S, Miyashita H, Kawashima M, Kawakita T, et al. (2010) The anti-oxidative role of ABCG2 in corneal epithelial cells. Invest Ophthalmol Vis Sci 51: 5617-5622.

\section{Acknowledgements}

This study was supported in parts by Minnesota Medical Foundation Grant, a grant from the Minnesota Lions Club, an unrestricted grant from the Research to Prevent Blindness, and a grant from the Undergraduate Research Opportunities Program at the University of Minnesota. 